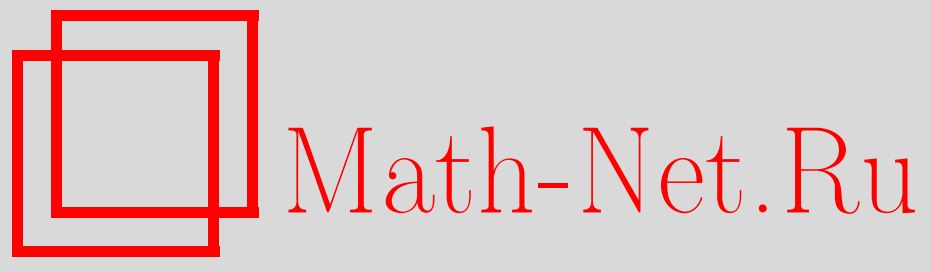

Д. Н. Туляков, Об одном свойстве условно сходящихся рядов, Матем. заметки, 2007, том 81, выпуск 2, 317-320 DOI: https://doi.org/10.4213/mzm3559

Использование Общероссийского математического портала Math-Net.Ru подразумевает, что вы прочитали и согласны с пользовательским соглашением http://www . mathnet.ru/rus/agreement

Параметры загрузки:

IP: 52.6 .47 .48

26 апреля 2023 г., 16:16:52

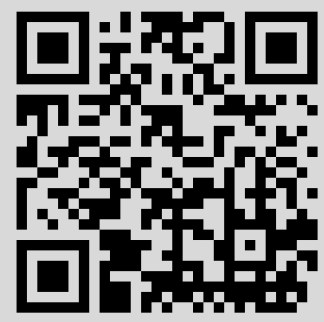




\section{Об одном свойстве условно сходящихся рядов}

\section{Д. Н. Туляков}

1. Введение. Хорошо известны существенные различия свойств абсолютно и условно сходящихся рядов (см. [1]-[3]). Для абсолютно сходящихся рядов следующее утверждение почти очевидно: если ряд $\sum a_{i}$ абсолютно сходящийся и $\left|a_{i}\right|<1$, то при всех натуральных $n$ ряды $\sum a_{i}^{2 n-1}$ (абсолютно) сходятся и их суммы при $n \rightarrow \infty$ стремятся к нулю. Из-за абсолютного характера сходимости это свойство сохранится при любых перестановках членов ряда. Возможно также ослабить условие на убывание $a_{i}$ : если для некоторого $k$ ряд $\sum a_{i}^{2 k}$ сходится, то суммы $\sum a_{i}^{2 n-1}$ стремятся к нулю.

Более интересно выполнение аналогичного свойства без каких-либо условий типа абсолютной сходимости, если модули чисел $a_{i}$ убывают монотонно. Из результатов о рядах из экспонент

$$
\sum_{k=1}^{\infty} a_{k} \exp \left(-\lambda_{k} z\right)
$$

(рядов Дирихле) следует (см. [4]), что требование

$$
\left|a_{i+1}\right| \leqslant\left|a_{i}\right| \quad \text { для любого } \quad i \in \mathbb{N}
$$

и факт существования всех сумм $\sum a_{i}^{2 n-1}$ влекут стремление этих сумм к нулю. В условно сходящихся рядах важен порядок слагаемых, поэтому неясно, насколько существенно требование монотонности. В связи с этим и другими вопросами сходимости рядов Дирихле Е. В. Щепиным был поставлен следующий

Вопрос. Пусть последовательность $\left\{a_{i}\right\}_{i=1}^{\infty}, a_{i} \in(-1,1)$, такова, что для всех $n \in \mathbb{N}$ ряд $\sum_{i=1}^{\infty} a_{i}^{2 n-1}$ условно-сходящийся. Обязательно ли

$$
\lim _{n \rightarrow \infty} \sum_{i=1}^{\infty} a_{i}^{2 n-1}=0 ?
$$

В рассматриваемой заметке показано, что ответ на этот вопрос отрицательный, более того, последовательность сумм нечетных степеней может иметь любое поведение.

2. Основная лемма и конструкция контрпримера. Сформулируем лемму, которая показывает, что суммы различных нечетных степеней вещественных наборов в достаточной степени независимы.

ЛЕмма 1. Для любого натурального $k$ и любого $\varepsilon>0$ существуют натуральное $N=$ $N(k, \varepsilon)$ и конечная последовательность $\left\{b_{i}\right\}_{i=1}^{N}$ вещественных чисел, для которых выполнены следующие два условия:

$$
\sum_{i=1}^{N} b_{i}^{2 k-1}=1,
$$

Работа выполнена при поддержке Российского фонда фундаментальных исследований (грант № 05-01-00697), программы "Ведущие научные школы" (грант № НШ-1551.2003.1), фонда ИНТАС NECCA (грант № 03-51-6637), программы № 1 ОМН РАН и Фонда содействия отечественной науке.

(C) Д. Н. Туляков, 2007 
для любого $n \in \mathbb{N} \backslash\{k\}$ и любого $M \in\{1, \ldots, N\}$

$$
\left|\sum_{i=1}^{M} b_{i}^{2 n-1}\right|<\varepsilon
$$

ЗАмечаниЕ. Из условия (2) при $n=1$ следует, что для всех $i \in\{1, \ldots, N\}\left|b_{i}\right|<2 \varepsilon$.

Покажем, как при помощи этой леммы строится пример бесконечной последовательности с нужными свойствами. Будем придавать $k$ последовательно натуральные значения, примем $\varepsilon=3^{-k}$ и обозначим через $N_{k}$ и $\left\{b_{i}^{(k)}\right\}$ полученные из леммы 1 значения. Теперь определим нужные нам числа $a_{i}$ следующим образом:

$$
a_{i+S_{k}}=b_{i}^{(k)}, \quad \text { где } S_{k}=\sum_{j=1}^{k-1} N_{j}, i \in\left\{1, \ldots, N_{k}\right\} \quad k \in \mathbb{N} .
$$

Легко видеть, что последовательность $\left\{a_{i}\right\}_{i=1}^{\infty}$ определяется таким способом однозначно и непротиворечиво. Убедимся в сходимости рядов $\sum_{i=0}^{\infty} a_{i}^{2 n-1}, n \in \mathbb{N}$, и оценим их суммы. Пусть $p<q$ - произвольные достаточно большие натуральные числа; обозначим через $k$ и $m$ такие числа, что $S_{k}<p \leqslant S_{k+1}, S_{m} \leqslant q<S_{m+1}$. Будем считать, что $k>n$. Разбивая сумму, по свойству (2) находим

$$
\begin{aligned}
\left|\sum_{i=p}^{q} a_{i}^{2 n-1}\right| & =\left|\sum_{i=1+S_{k}}^{q} a_{i}^{2 n-1}-\sum_{i=1+S_{k}}^{p-1} a_{i}^{2 n-1}\right| \\
& \leqslant\left|\sum_{i=1+S_{m}}^{q}\left(b_{i-S_{m}}^{(m)}\right)^{2 n-1}\right|+\sum_{r=k}^{m-1}\left|\sum_{i=1+S_{r}}^{S_{r+1}}\left(b_{i-S_{r}}^{(r)}\right)^{2 n-1}\right|+\left|\sum_{i=1+S_{k}}^{p-1}\left(b_{i-S_{k}}^{(k)}\right)^{2 n-1}\right| \\
& \leqslant 3^{-k}+\sum_{r=k}^{m} 3^{-r}<\frac{5 \cdot 3^{-k}}{2} .
\end{aligned}
$$

Следовательно, для всех $n \in \mathbb{N}$ ряд $\sum a_{i}^{2 n-1}$ сходится по признаку Коши. Для его суммы в силу (1), (2) и выбора $\varepsilon$ верна оценка

$$
\left|\sum_{i=1}^{\infty} a_{i}^{2 n-1}-1\right|=\left|\sum_{k=1}^{\infty}\left(\sum_{i=1}^{N_{k}}\left(b_{i}^{(k)}\right)^{2 n-1}\right)-\sum_{i=1}^{N_{n}}\left(b_{i}^{(n)}\right)^{2 n-1}\right|<\sum_{k(\neq n)} 3^{-k}<\frac{1}{2} .
$$

Поэтому $\sum_{i} a_{i}^{2 n-1}>1 / 2$, т.е. не стремится к нулю при $n \rightarrow \infty$.

3. Доказательство основной леммы. Прежде всего отметим, что для наших целей достаточно доказать вариант леммы, полученный заменой $=$ на $\geqslant$ в условии (1). Если такие $b_{i}$ найдены, то для достижения равенства в (1) достаточно их умножить на некоторую величину из интервала $(0,1)$, что не нарушает условие $(2)$. Поэтому будет доказан вариант леммы с условием $\geqslant 1$ в формуле (1).

Для дальнейшего нам понадобится следующий факт из элементарной математики (вполне возможно, что у него есть более простое доказательство, чем приведено ниже).

ЛЕмма 2. Пусть $k$ - натуральное число. Тогда найдутся вещественные $x_{1}, x_{2}, \ldots$, $x_{2 k-1}$ maкue, что

$$
\sum_{i} x_{i}^{2 k-1}=1 \quad u \quad \text { npu } n \in \mathbb{N}, n<k \quad \sum_{i} x_{i}^{2 n-1}=0 .
$$


ДоказАтельство. Рассмотрим отображение $F: \mathbb{R}^{k} \rightarrow \mathbb{R}^{k}, F(\vec{x})=\vec{y}$, задаваемое формулами $y_{j}=\sum_{i} x_{i}^{2 j-1}$. Его производная в точке $(0,1, \ldots, k-1)$ невырождена, поэтому оно обратимо в окрестности этой точки. Следовательно, система уравнений

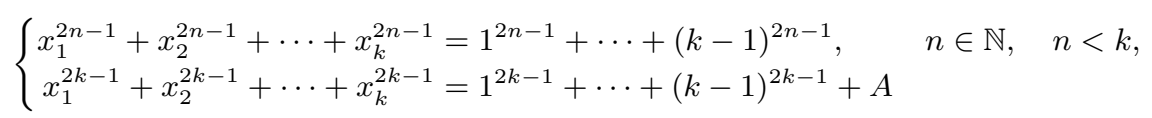

в окрестности значения $A=0$ имеет вещественное решение. Возьмем некоторое $A>0$, для которого система имеет вещественное решение, дополним набор $\left(x_{1}, \ldots, x_{k}\right)$ по формулам $x_{k+n}=-n, n<k$, затем полученный набор длины $2 k-1$ поэлементно разделим на $\sqrt[2 k-1]{A}$. Полученный набор удовлетворяет условиям (3).

Вернемся к доказательству основной леммы. Построим числа $b_{i}$ с нужными свойствами, группируя их по $2 k-1$ штук, по следующим формулам:

$$
b_{(2 k-1) s+i}=C\left(M_{1}+s\right)^{-1 /(2 k)} x_{i} \quad \text { для } i \in\{1, \ldots, 2 k-1\}, \quad s \in \mathbb{N}, 0 \leqslant s<M_{2},
$$

где $x_{i}$ - числа из леммы 2 , а константы $C, M_{1}, M_{2}$ будут определены ниже. В данном случае $N=(2 k-1) M_{2}$. Оценим суммы $\sum b_{i}^{2 n-1}$ отдельно для трех различных случаев: $n<k, n=k, n>k$. Обозначим через $H$ величину $H=\max _{i}\left|x_{i}\right|$.

Случай $1(n<k)$. Возьмем произвольное $M \in\{1, \ldots, N\}$, и пусть $M=(2 k-1) m+r$, где $m=[(M-1) /(2 k-1)]$. Тогда

$$
\begin{aligned}
\left|\sum_{i=1}^{M} b_{i}^{2 n-1}\right|< & \sum_{s=0}^{m-1}\left|\sum_{i=1}^{2 k-1} b_{(2 k-1) s+i}^{2 n-1}\right|+\left|\sum_{i=1}^{r} b_{(2 k-1) m+i}^{2 n-1}\right| \\
= & \sum_{s=0}^{m-1} C^{2 n-1}\left(M_{1}+s\right)^{-(2 n-1) /(2 k)}\left|\sum_{i=1}^{2 k-1} x_{i}^{2 n-1}\right| \\
& +C^{2 n-1}\left(M_{1}+m\right)^{-(2 n-1) /(2 k)}\left|\sum_{i=1}^{r} x_{i}^{2 n-1}\right| \\
< & 0+(2 k-1)(C H)^{2 n-1} M_{1}^{-(2 n-1) /(2 k)} .
\end{aligned}
$$

Случай $2(n=k)$. В этом случае нас интересует сумма $b_{i}^{2 k-1}$ для всех $i$. Тогда

$$
\begin{aligned}
\left|\sum_{i=1}^{N} b_{i}^{2 k-1}\right| & =\left|\sum_{s=0}^{M_{2}-1} \sum_{i=1}^{2 k-1} b_{(2 k-1) s+i}^{2 k-1}\right| \\
& =\left|\sum_{s=0}^{M_{2}-1} C^{2 k-1}\left(M_{1}+s\right)^{-(2 k-1) /(2 k)} \sum_{i=1}^{2 k-1} x_{i}^{2 n-1}\right| \\
& =C^{2 k-1} \sum_{m=M_{1}}^{M_{1}+M_{2}-1} m^{-(2 k-1) /(2 k)} \\
& =2 k C^{2 k-1}\left(\sqrt[2 k]{M_{1}+M_{2}}-\sqrt[2 k]{M_{1}}+o\left(M_{1}^{-(2 k-1) /(2 k)}\right)\right) .
\end{aligned}
$$

Случай $3(n>k)$. Возьмем произвольное $M \in\{1, \ldots, N\}$, и пусть $M=(2 k-1) m+r$, где $m=[(M-1) /(2 k-1)]$. Тогда

$$
\begin{aligned}
\left|\sum_{i=1}^{M} b_{i}^{2 n-1}\right| & <\sum_{s=0}^{m-1}\left|\sum_{i=1}^{2 k-1} b_{(2 k-1) s+i}^{2 n-1}\right|+\left|\sum_{i=1}^{r} b_{(2 k-1) m+i}^{2 n-1}\right| \\
& <(2 k-1)(C H)^{2 n-1} \sum_{m=M_{1}}^{M_{1}+M_{2}-1} m^{-(2 n-1) /(2 k)}
\end{aligned}
$$




$$
\begin{aligned}
& <(2 k-1)(C H)^{2 n-1} \int_{M_{1}-1}^{\infty} m^{-(2 n-1) /(2 k)} d m \\
& =\frac{2 k(2 k-1)}{2 n-2 k-1}(C H)^{2 n-1}\left(M_{1}-1\right)^{-(2 n-2 k-1) /(2 k)} .
\end{aligned}
$$

Считая $\varepsilon<1$, возьмем $C=1 / H, M_{1}=1+(2 k(2 k-1))^{2 k} \varepsilon^{-2 k}$. Тогда для случаев 1 и 3 получим оценку сумм: $<\varepsilon$ при всех подходящих $n$. Поскольку в случае 2 оценка снизу неограниченно растет при $M_{2} \rightarrow \infty$, то можно выбрать $M_{2}$ так, что левая часть будет больше единицы, что и требовалось доказать.

\section{СПИСОК ЦИТИРОВАННОЙ ЛИТЕРАТУРЫ}

[1] Б. Гелбаум, Дж. Олмстед, Контрпримерь в анализе, Мир, М., 1967. [2] W. Rudin, Real and Complex Analysis, McGraw Hill, New York, 1987. [3] Г. Харди, Расходящиеся ряды, Изд-во иностранной литературы, 1951. [4] А. Ф. Леонтьев, Рядъ экспонент, Наука, M., 1976.

\section{Д. Н. Туляков}

Поступило

Институт прикладной математики им. М.В. Келдыша РАН

18.07.2006 